\title{
UNRAVELLING THE METABOLIC PROGRESSION OF BREAST CANCER CELLS TO BONE METASTASIS BY COUPLING RAMAN SPECTROSCOPY AND A NOVEL \\ USE OF MCR-ALS ALGORITHM
}

\author{
Monica Marro ${ }^{a,{ }^{*}}$, Claudia Nieva ${ }^{b}$, Anna de Juan ${ }^{c}$, and Angels Sierra ${ }^{d, e,{ }^{* *}}$ \\ ${ }^{a}$ ICFO- ICFO-Institut de Ciencies Fotoniques, The Barcelona Institute of Science and Technology, 08860 Castelldefels, \\ Barcelona, Spain \\ ${ }^{b}$ IDIBELL-Institut d'Investigació Biomèdica de Bellvitge, Av. Castelldefels, Km 2.7, 08907 L'Hospitalet de Llobregat, Barcelona, \\ Spain \\ c Department of Chemical Engineering and Analytical Chemistry, Universitat de Barcelona. Diagonal 645, 08028 Barcelona, \\ Spain

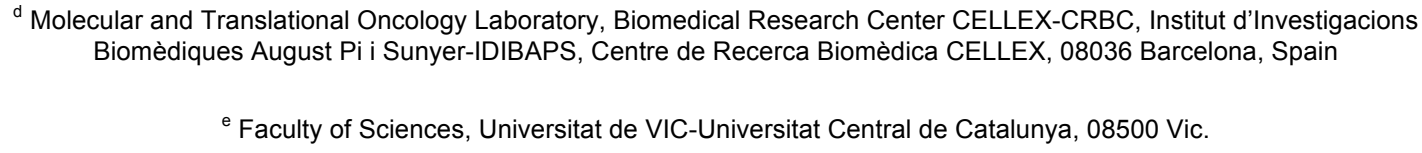

${ }^{\mathrm{e}}$ Faculty of Sciences, Universitat de VIC-Universitat Central de Catalunya, 08500 Vic.

Email: *: monica.marro.san@gmail.com, monica.marro@icfo.eu, www.icfo.eu; **: asierrajim@gmail.com; masierra@clinic.ub.es; http://www.hospitalclinic.org/. http://www.idibaps.org/

\begin{abstract}
Raman spectroscopy (RS) has shown promise as a tool to reveal biochemical changes that occur in cancer processes at the cellular level. However, when analysing clinical samples, RS requires improvements to be able to resolve biological components from the spectra. We compared the strengths of Multivariate Curve Resolution (MCR) versus Principal Component Analysis (PCA) to deconvolve meaningful biological components, formed by distinct mixtures of biological molecules, from a set of mixed spectra. We exploited the flexibility of the MCR algorithm to easily accommodate different initial estimates and constraints. We demonstrate the ability of MCR to resolve undesired background signals from the RS that can be subtracted to obtain clearer cancer cell spectra. We used two triple negative breast cancer cell lines, MDA-MB 231 and MDA-MB 435, to illustrate the insights obtained by RS that infer the metabolic changes required for metastasis progression. Our results show that increased levels of amino acids and lower levels of mitochondrial signals are attributes of bone metastatic cells, whereas lung metastasis tropism is characterized by high lipid and mitochondria levels. Therefore, we propose a method based on the MCR algorithm to achieve unique biochemical insights into the molecular progression of cancer cells using RS.
\end{abstract}

\section{INTRODUCTION}

Metastatic cancer cells are characterized by reprogramming of the cellular metabolism that leads to increased uptake of glucose for use as an anabolic and catabolic substrate, thus satisfying the increased demands for macromolecules and energy for proliferation ${ }^{1}$. Recent investigations into the mechanisms that underlie the Warburg effect, also known as aerobic glycolysis, suggested that mitochondrial uncoupling can promote aerobic glycolysis, which may represent a shift to the oxidative metabolism of non-glucose carbon sources ${ }^{2}$. Understanding and analysing mitochondrial uncoupling and the metabolic changes observed in cancer cells are important because they may be associated with increased resistance to chemotherapeutic insults ${ }^{3}$. Moreover, increased mitochondrial oxidative metabolism may be a key driver of tumour cell metastasis ${ }^{4}$.

We have previously studied the biochemical characteristics of breast cancer metastatic cells by Raman spectroscopy (RS) ${ }^{5}$, a technique based on the inelastic dispersion of monochromatic radiation that provides specific vibrational signatures of chemical bonds and therefore can be used to determine the chemical composition of cells ${ }^{6}$. RS has been used in the past decade to study 
biological samples with the help of statistical analysis. Various techniques have been used to find groups and clusters in the RS data set, such as Principal Component Analysis $(\mathrm{PCA})^{5,7,8}$, K-means clustering ${ }^{9,10}$, and Neural networks ${ }^{11}$. Partial-Least-Squares Discriminant Analysis (PLS-DA) allows the construction of discriminatory models to distinguish different types of samples and thus it has been used to differentiate breast cancer metastatic cells from non-metastatic cells without a basal-like phenotype ${ }^{5}$ Twodimensional correlation has been used to gain insights into the Raman bands and band evolution such as broadening, shifts, etc. ${ }^{12}$ to study dynamic processes. However, the multivariate techniques mentioned above do not provide sufficient information to extract the molecular picture of the process being analysed and thus do not exploit all the capabilities of RS. Unmixing techniques such as Vertex Component Analysis (VCA) ${ }^{10,13,14}$ has been used to analyse Raman spectral data in an effort to resolve more "pure" signals. Although VCA and N-FINDR have been often used, these algorithms assume the presence of "pure" pixels in the data set (which in the case of Raman spectra of biomedical samples is very complicated due to the highly mixed molecular content present). Second, they need to use necessarily non-negativity and closure constrains to work, which is not always possible if Raman spectra are transformed, e.g., into derivative spectra, or when pixel-to-pixel high variations of total signal exist.. Within the group of unmixing algorithms, Multivariate Curve Resolution-Alternating Least Squares (MCR-ALS), is a very flexible algorithm in which many constraints can be easily included, like non-negativity, selectivity or local rank ${ }^{15,25}$, information of presence/absence of components in different data blocks in multiset structures, (so-called correspondence among species ${ }^{22}$ ). However, all constraints can be optionally applied in MCR and none of them is compulsory. In this paper, we used non-negativity in the concentration and spectral direction and the selectivity constraint linked to spectra of background contributions in the MCR algorithm in order to resolve more easily meaningful spectral profiles of biological components from the mixed Raman spectra of cancer cells.

There are various drawbacks that need to be overcome to fully exploit RS for diagnostic purposes ${ }^{12}$. First, cells are grown on the surface of glass materials and the focus of the laser needs to be close to or past the surface window. Thus part of the confocal volume analysed includes a portion of the glass, which consequently produces undesired background signals in the RS. Second, samples contain stains and are prepared on cover slips that give a very high signal and disturb the inherent cellular RS. Bonnier et al.

${ }^{17}$ tried to remove the effect of the background signals in the spectra by growing the cells in collagen matrices on the top of coverslips but this requires sample processing and modification of already established biomedical protocols. Finally, in applications for in-vivo RS analysis which involve fiber optical probes, high background signals from silica perturb the Raman spectra and consequently reduce the ability to perform stable and robust statistical models. Therefore, further development is essential to reduce background spectral signals in these cases ${ }^{18,19,20}$.

In this study, we present a powerful statistical methodology to remove undesired background signals and extract meaningful molecular components from Raman spectra. The method is based on the use of MCR-ALS, a very flexible and powerful algorithm that has been applied to several chemical problems ${ }^{21}$ Recently, it has been applied to biomedical RS data ${ }^{22,23,24,25}$. Specifically, we first compared the ability of PCA and MCR analysis applied to the same RS data to resolve molecular spectral contributions. Second, the use of the flexibility of the MCR-ALS algorithm was used to include specific constrains (non-negativity and selectivity constraint) to extract the quartz and water background signal from the spectral data and remove it from the RS. Both procedures have been used to gain insights into the metabolic changes that breast cancer cells undergo while progressing with different metastasis tropisms ${ }^{26}$.

We used two triple negative human breast cancer cell lines, MDA-MB-231 and its osteotropic cell line B02 subclone and MDAMB-435 (435P) with tropism to metastasize in the lung and its bone metastatic variant 435-B. We carried out RS and MCR-ALS analysis to study biochemical differences between metastasis tropisms. The results showed that an increase in amino acids and a 
decrease in mitochondrial signals were attributes of bone metastatic cells, whereas lung metastasis tropism was characterized by high lipid and mitochondria (cytochrome C and RNA) levels. We demonstrate that RS coupled with the MR-ALS algorithm resolve meaningful molecular components during metastatic progression of cancer cells, giving more biochemical information than other commonly used mathematical techniques. Furthermore, we propose exploiting the flexibility of the MCR-ALS algorithm to enhance and expand the strengths of RS for clinical diagnosis, and demonstrate the successful subtraction of undesired background signals (that are unavoidable in clinical practice), allowing the extraction of more valuable information from RS.

\section{MATERIALS AND METHODS}

\section{Cell lines used}

We used the breast cancer bone metastatic cell line MDA-MB-231 (231P), originally obtained from the European Type Culture Collection (ECACC 92020424), and B02 (kindly provided by Philippe Clezardin, INSERM, UMR 664, IFR62, Laennec School of Medicine, F-69372, Lyon, France) established from bone metastases caused by MDA-MB-231 after six in vivo/in vitro passages in nude mice using a heart injection mode $\mathrm{l}^{27}$. The proprietary $435-\mathrm{B}$ cells were obtained from primary cultures of bone metastasis induced by MDA-MB 435 (435P) cells inoculated in the intramammary fat path ${ }^{28}$. The proprietary B02/PRDX2 cells ${ }^{29,30,31}$ were $^{2}$ previously obtained from highly bone metastatic BO2/GFP cells overexpressing PRDX2 (BO2/PRDX2) through the pBIL vector system.

Cells were cultured under standard conditions in DMEM/F12 medium supplemented with 5\% horse serum, $1 \mathrm{mM}$ pyruvate, $2 \mathrm{mM}$ L-glutamine in $5 \% \mathrm{CO}_{2}-95 \%$ air at $37^{\circ} \mathrm{C}$ in a humidified incubator.

\section{Raman spectroscopy (RS)}

For RS analysis $3 \cdot 10^{5}$ cells were used and measurements were carried out by seeding cells over a quartz crystal (ESCO products, Oak Ridge, NJ) in six-well plates (Becton Dickinson, NJ). After 24 h, cells were fixed with 4\% cold paraformaldehyde (PFA) in PBS1x for 15 min, washed with PBS 1x and maintained in the same solution at $4^{\circ} \mathrm{C}$ until the spectroscopic analysis.

A total of 225 spectra were obtained from the cell cytoplasm (few $\mu \mathrm{m}$ away from the nucleous, see the inset in Fig $2 \mathrm{~b}$ ), from both the parental cell lines and their corresponding metastatic variants.

The Raman system used was an inVia Renishaw (Apply Innovation, Gloucestershire, UK) and comprises a $532 \mathrm{~nm}$ laser that supplies an excitation beam of about $10 \mathrm{~mW}$ power, which is focused onto the sample via a microscope with a $60 \mathrm{x}$ objective (Edmund, York, UK) using a backscattered configuration. The laser spot size in the sample is $0.8 \mu \mathrm{m}$. The Raman spectrum is recorded on a deep depletion charge-coupled device (CCD) detector (Renishaw RenCam). The recorded Raman spectrum is digitalized and displayed on a personal computer using Renishaw WiRE software, which allows the experimental parameters to be set. The spectra were background subtracted with a custom-written Labview program using an established method ${ }^{32}$.

\section{Statistical analysis}

First, an exploration of the spectral dataset was performed using PCA. The number of components selected was the number in which the cumulative variance explained was more than $99 \%$. Two independent analyses (PCA and MCR) were performed on the same dataset, which contained the spectra of the cell lines in rows. Additionally, to improve background component resolution five spectra from the PBS solution on the same quartz substrate and at the same focal distance as the cell measurements and far away from the cells were added. These spectra were used to include selectivity constraints to the MCR algorithm indicating the presence/absence of molecular components in some of the measured spectra. For the MCR-ALS algorithm, the protocol followed was: first, the initial estimates were selected using a purest variable selection method algorithm, which selects the purest rows (spectra) in the dataset; second, the iterative least squares calculation started with the addition of constraints (non-negativity of 
spectra and concentration matrices and selectivity constraint for concentrations linked to spectra with only background

contributions). Finally, the quality parameters from the MCR model were calculated: Lack of fit:

$$
L O F=100 \sqrt{\frac{\sum_{i=1}^{n} \sum_{j=1}^{m} e_{i, j}^{2}}{\sum_{i=1}^{n} \sum_{j=1}^{m} d_{i, j}^{2}}} \text { and }
$$

\[ R^{2}=100 \frac{\sum_{i=1}^{n} \sum_{j=1}^{m} d_{i, j}^{2}-\sum_{i=1}^{n} \sum_{j=1}^{m} e_{i, j}^{2}}{\sum_{i=1}^{n} \sum_{j=1}^{m} d_{i, j}^{2}} \text { where } e_{i, j}=d_{i, j}-\hat{d}_{i, j} \text { and } \mathrm{d}_{\mathrm{i}, \mathrm{j}} \text { is the original data matrix and } \hat{d}_{i, j} \]
the parameter:

the calculated by means of the MCR-ALS algorithm.

For PCA and MCR analysis two different Matlab toolboxes were used: PLS toolbox (from Eigenvector Research) and MCR-ALS toolbox (from University of Barcelona).

\section{RESULTS AND DISCUSSION}

Multivariate Curve Resolution-Alternating Least Squares (MCR-ALS) is able to resolve more meaningful molecular components than Principal Component Analysis (PCA) on Raman spectra (RS)

First, a PCA was performed on the spectral data matrix containing 135 spectra from the cytoplasm area (avoiding the nucelous) of 231, B02, 435 and 435-B cells and five more from PBS, from which the principal component loadings were plotted (Fig. 1A). These results were compared with those obtained from the MCR-ALS algorithm subjected to specific constraints (non-negativity of spectra and concentrations, and selectivity constraint) (Fig. 1B, 1C) and applied to the same spectral dataset. We demonstrated that when using MCR-ALS more chemically meaningful molecular components are extracted. Furthermore, we were able to separately resolve two components containing quartz and water signals.

The results of PCA (Fig. 1A) showed that the signals of different substrates were mixed in among the loadings of the principal components. The contributions of quartz signal ${ }^{17,33}$ (mainly bands around 800 and $1050 \mathrm{~cm}^{-1}$ ) were found in PC3, together with water signal and other cellular substrates. Indeed, the presence of positive and negative bands in the principal component loadings made the assignment of each component to a molecular RS contribution difficult. In contrast, MCR decomposed four meaningful components (Fig. 1B) that could be assigned to cellular lipids (component 1), quartz signal (component 2), water signal ${ }^{31}$ (component 3) and proteins and other cellular components (component 4). The variance explained by PCA and MCR models was $99.74 \%$ and $99.66 \%$, respectively.

MCR spectra provided more chemically meaningful results than PCA, providing two components, 2 (quartz) and 3 (water), that did not correspond to cellular molecular components ${ }^{17,33,34}$. Indeed, the higher ability of MCR to resolve pure signals relied on the flexibility of the MCR-ALS algorithm. First, the spectral data matrix was constructed by displaying all cell RS in rows and adding five more rows containing the signal from PBS, acquired at the same focal distance as for the cells on the quartz substrate. Second, a purest variable selection algorithm was used to find the purest spectra in the dataset in order to provide initial estimates of the MCRALS algorithm. As a result the four most dissimilar spectra found were: two signals from PBS solution and two from different cell lines. Once the initial estimates were selected, the iterative least square calculation was initialized by placing constraints on the algorithm. First, we included non-negativity in the resolved spectra and concentration profiles. Second, a matrix showing the presence/absence of molecular components such as water, quartz and cellular spectra was encoded (Fig.1c). In the rows where there was absence of cellular spectra, zeros were included to the components from Quartz and Water that we wanted to resolve. The above process yielded four chemically and physically meaningful components. 
Unlike MCR, PCA found the orthogonal directions in the dataset with the maximum variance, but no additional chemical information could be added. Consequently, in the case of complicated biological Raman datasets, PCA could be used for an exploratory analysis of the dataset to find for instance outliers or groups or to understand the chemical structure of the data as a first approach, but not meaningful molecular spectral components can be unmixed using this method. This problem has been mentioned in previous cellular studies ${ }^{35}$ and in studies involving image segmentation ${ }^{33}$.

\section{Subtraction of the background signals by MCR allowed the resolution of metabolites in metastatic breast cancer cells}

In order to clean and remove quartz and water signals from the original spectral dataset, direct subtraction of both signals (MCR loadings) multiplied by the concentration matrix (MCR scores) of those components for each original spectrum was performed. This led to greater visibility of some spectral changes inherent to the cellular spectra, making it easier to build a statistical model with chemically meaningful outputs. The results are shown in Figure 2, where the plots of spectra before (Fig. 2A) and after (Fig. 2B) the subtraction of quartz and water signals are compared. Furthermore, by using this method, statistical analysis of cell RS is improved as long as the background is removed. Thus, based on the clean cell RS, a new MCR model was calculated to resolve pure molecular RS involved in the different biochemical components of metastatic progression (Fig. 3).

Recently, various applications of MCR to Raman images have detected the contribution of background signals in the resolved components $^{36,37,38,39}$. However, to our knowledge this is the first time that the MCR-ALS algorithm has been proposed as a tool to subtract the background signal contributions in the Raman analysis of cells when using standard sample preparation. For clinical relevancy and acceptance of RS by the clinical community, it is important that the sample preparation protocols necessary for RS are coincident with current practice. Nowadays this is a challenging issue and researchers are working to overcome this limitation. For instance, a study compared Raman signals of unprocessed tissue preparations and dewaxed tissue sections, demonstrating that tissue processing has a significant impact on the extracellular structure, in particular on the lipidic structure ${ }^{40}$. Also, in the clinical applications of RS related with endoscopy, background signals due to the use of fiber optics have been reported as one of the main issues $^{41,19}$. Furthermore, the use of routine stained tissue sections or cells for RS analysis has been limited due to background signals that interfere with the Raman spectra ${ }^{42}$. Therefore, to prevent the loss of critical cellular information, RS should be compatible, as much as possible, with conventional sample preparation, without the need for further processing. This challenge might be overcome by resolving non-inherent signals from current cytological preparations from the cell spectra. In this way, everyday techniques could be performed in parallel with Raman spectroscopy analysis using the same samples. Moreover, the use of stored samples might permit retrospective studies based on prognosis and patient follow-up.

It is important to note that from a matrix with very similar cell RS the MCR-ALS algorithm permitted the resolution of three meaningful molecular components. Indeed, RS were assigned to almost pure molecules. Therefore, this analytical method extracted more biochemical information than other commonly used techniques, such as PCA or cluster analysis, providing information about the biochemical changes occurring in metastatic cancer cells (Fig. 3). The concentrations of each MCR component for each cell variant measured were plotted (Fig. 3 right) with their corresponding spectra (Fig. 3 left). In particular component 1 was assigned to cell lipids ${ }^{43}$. Raman bands in component 2 have been related to amino acids and cell proteins ${ }^{44,45}$. Finally, component 3 contained bands previously assigned to mitochondria, containing bands from cytochrome $\mathrm{C}^{46-54}$ and DNA or nucleotides ${ }^{55}$. As mitochondria are the organelles in the cytoplasm that contain the highest concentrations of DNA and cytochrome C, this led to the conclusion that component 3 could be used as a measure of the number of mitochondria present in breast cancer metastatic cells. Table 1 shows the main assignments of Raman bands from each component. 
When comparing the three main biochemical components that differentiate cells, we observed that component 1 , assigned to cell lipids, was inversely proportional to capacity for bone metastasis such that its value decreased as long as the bone tropism increased. This is in agreement with the described "low-lipid" phenotype that characterized breast cancer cells compared with normal breast epithelial cells, and negatively correlated with invasiveness and metastatic progression ${ }^{56}$. The lowest level of component 1 was found in B02 cells and the highest level was found in 435P cells, which showed the greatest lung metastatic tropism. Moreover, we were able to distinguish two different populations belonging to 435P cells, one with lower lipid content that was clearly enriched in the 435-B bone metastatic variant (Fig. 3A). These clear differences in lipid level between the two triple negative (231P and 435P) breast cancer metastatic cell lines might be due to the lung metastatic background that characterizes $435 \mathrm{P}$ cells, in contrast to $231 \mathrm{P}$ cells that have greater bone metastatic ability. Therefore, the low fat content is associated with bone metastatic ability.

Component 2, which was assigned to protein content, increased in parallel with increasing bone metastasis tropism. The lowest level of component 2 was found in 435P cells, and the highest level was found in the B02 bone metastatic variant. Moreover, 435-B had a higher amino acid content than 435P (Fig. 3B).

Component 3, which contain bands from cytochrome $\mathrm{C}^{46-54}$ and DNA or nucleotide bands ${ }^{55}$, was inversely related to bone metastatic ability. Thus, similarly to lipid content, scores in Component 3 increased in 435P cells with regard to bone metastatic ones (Fig. 3C). In summary, the MCR-ALS algorithm applied to a set of cytological RS provided insights into the subtle metabolic changes that breast cancer cells undergo on the path towards bone metastasis tropism, as revealed by the different profiles obtained in the analysis of $231 \mathrm{P}$ cells, with tropism to metastasize in bone, and 435P, with tropism to metastasize in lung. Moreover, the respective bone metastatic variants, B02 and 435-B, were classified according to their corresponding phenotype. These results are consistent with reports demonstrating that the mechanism used to cope with the cumulative reactive oxygen species differs between lung and bone tissues, with the lower oxygen gradient in bone marrow inducing fewer metabolic free radical challenges ${ }^{54}$.

Mitochondria are an important cell metabolic hub because they orchestrate the biosynthesis of lipids, proteins, and nucleic acids and maintain redox homeostasis via the generation of NADH and NADPH ${ }^{5}$. Although in normal cells the TCA cycle is mostly involved in glucose oxidation, the mitochondrial metabolism of cancer cells is generally uncoupled from glycolysis ${ }^{58}$. It has been reported that mitochondrial glucose oxidation may be incompatible with the survival of some cancer cells ${ }^{59}$. Due to genetic and environmental cues, such as hypoxia, the enzyme responsible for the entry of glucose-derived carbons into the mitochondria, the pyruvate dehydrogenase complex $(\mathrm{PDH})$, is frequently inactivated in cancer ${ }^{60}$. This mechanism might be particularly important in metastatic cells in an hypoxic organ like bones. Furthermore, the homeostatic mechanism of the antioxidant system might prevent collapse by redirecting the glycolytic flux into the pentose phosphate pathway ${ }^{58}$. All together, these results clearly indicate that a metabolic switch in breast cancer cells might be used to fit with the microenvironment at the metastatic loci.

\section{RS supported by MCR-ALS algorithm dissects metabolic functions of metastatic breast cancer cells}

Since breast cancer bone metastatic cells might be characterized by a clear increase in protein content and a lower lipid content than other breast cancer cells, we analyzed B02 cells overexpressing PRDX2 (B02/PRDX2 cells) that exhibited a low bone metastatic burden in vivo ${ }^{29}$. PRDX2 is a highly efficient redox protein that neutralizes hydrogen peroxide, resulting in protection of cells from oxidative damage and in regulation of peroxide-mediated signal transduction events ${ }^{62}$. The low metastatic burden of B02/PRDX2 cells in bones suggests that PRDX2 might interfere in the preferential bone metastatic cells' metabolism with deleterious consequences for carcinoma cells that try to adapt to the bone microenvironment. This model was included in the study to better assess the sensitivity of RS-MCR to differentiate small molecular changes that induce important functional cell changes. 
The model described above was tested in an independent matrix containing the RS of BO2, 231P and B02/PRDX2 cells. First, the signals from quartz and water were subtracted following the method described before and then an MCR was built to confirm the molecular behavior of the different cell lines and specifically the role of PRDX2 up-regulation Fig. 4.

MCR analysis of these experiments revealed that lipid levels were higher in B02/PRDX2 cells as reflected in Component 1 levels (Fig. 4 A) and strongly correlated with the above observation that the lipid content of cells was inversely related to the bone metastasis potential (Fig. 4 A). In fact, as previously demonstrated ${ }^{63}$, the preferential bone metastatic metabolism of B02 cells can be disrupted by overexpressing PRDX2, which induces low glucose dependence. These results suggest a metabolic shift from glucose oxidation to fatty acid oxidation that would resist metabolic insults in an hypoxic and hypoglycemic tissue like bones.

Moreover, Component 2 (Fig. 4 B) (related to amino acids) was found at lower levels in BO2/PRXD2 cells in comparison to B02 cells, but at similar levels to those in MDA-MB-231 cells, supporting the idea that lower amino acid content is associated with higher bone tropism. In addition, Component 3 was found at higher levels in BO2/PRXD2 cells, coinciding with the fact that cytochrome $\mathrm{C}$ was previously found to be inversely associated with bone tropism, increased from $\mathrm{BO} 2$ to $\mathrm{BO} 2 / \mathrm{PRXD} 2$ cells that is not able to metastasize in bones (Fig. 4 C). This is in agreement with the results showed in Fig 3 C, where we defined bone metastasis tropism, and which all together supported the biological use of MCR applications to sensitively analyze the fine biochemical changes that occur in bone metastasis progression. Moreover, a PLS-DA analysis to discriminate BO2 cells from MDA-MB-435P was performed (Fig. 5) that achieved a specificity of $100 \%$ and specificity of $90 \%$. Cross-validation was performed using 10 samples that were excluded from the dataset. This corroborates our hypothesis and highlighted that RS is able to assess and identify bone tropism in breast cancer cells

Despite advances in treatment, $20 \%$ to $30 \%$ of patients with early breast cancers will experience relapse with distant metastatic disease $^{63}$. Triple negative tumours have the highest metastatic rate with regard to luminal and HER 2 positive tumors, with bone and lung being the main target of breast cancer metastasis. Transcriptomic analysis of a variety of cell lines has identified the genes that mediate metastasis to bone or lungs ${ }^{64,65,66}$, but in clinical settings breast cancer subtyping is based on pathological characteristics ${ }^{67}$. With the development of metabolomics we have learnt that metabolic variability and flexibility enable tumour cell progression by generating ATP as an energy source ${ }^{68}$. Three major components of the metabolic transformation of cancer cells have been $\operatorname{described}^{69}$ : aerobic glycolysis, mitochondrial metabolism, and lipid synthesis.

We provide data demonstrating that RS coupled with the MCR-ALS algorithm is a powerful method that can be used in a clever and flexible way to reveal the underlying molecular information stored in RS, allowing a further step forward in the analysis of biological RS relative to the multivariate techniques already used to analyse metabolic changes in cancer cells. Moreover, RS provides a unique possibility for real-time and non-intensive-processing of tissue material for the detection of normal, precancerous and cancerous breast tissues ${ }^{70}$. We propose the use of the MCR-ALS algorithm to enhance and expand the strengths of RS for clinical diagnosis.

\section{CONCLUSION}

We propose a new methodology for extracting the useful molecular information encoded in RS of biological samples. By using the flexible MCR-ALS algorithm meaningful molecular components can be extracted, thanks to the prior subtraction of background signals that perturb the inherent cell RS and including specific constrains indicating the presence/absence of molecular components. This methodology opens up new possibilities for the clinical diagnostic use of RS, since it permits the removal of signals from substrates or chemicals used in cytological techniques. It also provides a rapid, reliable and label free method with which to 
disentangle, in cancer cytology, the biochemical components involved in metastasis progression, and as such could improve prognosis and early prevention.

We applied this methodology to study the molecular differences between primary breast cancer cell lines and their metastatic variants in bone. We identified biochemically meaningful molecular components that play a role in the metastatic ability of breast cancer cells. Specifically, the protein component was found at higher levels whereas mitochondria and lipids bands were found at lower levels in bone metastatic cell variants (B02) in relation to their corresponding parental cell lines (231P and 345P). Thus, a ratio of these three components might be used to validate this data in clinical samples. In addition, a PLS-DA was performed showing high sensitivity and specificity in the discrimination of $\mathrm{BO} 2$ and MDA-MB-435P cells.

\section{ACKNOWLEDGMENTS AND FUNDING}

This study was supported by grants from the Spanish Ministry of Health and Consumer Affairs FIS-PI14/00336, both from the I+D+I National Plan with financial support from ISCIII-Subdirección General de Evaluación and the Fondo Europeo de Desarrollo Regional (FEDER), and by grants from 2014 SGR 530 and from the Fundació Privada Cellex.

Dr. Marro acknowledges financial support from the Spanish Ministry of Economy and Competitiveness through the "Severo Ochoa" program for Centres of Excellence in R\&D (SEV-2015-0522), from Fundació Privada Cellex, Fundación Mig-Puig, from Generalitat de Catalunya through the CERCA program and from Laserlab-Europe (EU-H2020 654148). This research has been partially conducted at ICFO's Super Resolution Light Microscopy and Nanoscopy Facility.

The authors are grateful to Toffa Evans from Assessorament Lingüístic i Terminologia Universitat de Barcelona for language advice and to the COST action BM1401, Raman4Clinics, https://www.raman4clinics.eu/raman4clinics-a-european-cost-action/.

\section{FIGURES}

Figure 1. MCR-ALS analysis gives more chemically meaningful components than PCA. PCA (A) and MCR-ALS (B) loadings of the matrix spectral dataset containing the Raman spectra (RS) obtained from the cytoplasm of cancer cell lines $231 \mathrm{P}$, B02, 435P and 435B and PBS. (C) MCR-ALS constraints were applied to the data: non-negativity and selectivity constraint to resolve quartz and PBS spectra.

Figure 2. Subtraction of background signals in cellular RS using MCR-ALS algorithm: RS before (A) and after the subtraction of quartz and water signals (B). MCR loadings from Figure 1B were used to resolve the meaningful molecular contributions. The figure inset in (B) shows an image of a MDA-MB-231 cell indicating with green arrows the areas in which the Raman spectra was obtained. A Raman image shows the lipid distribution (Component 1) in the cytoplasm of the cell.

Figure 3. Using MCR-ALS analysis of Raman spectra from 231P, B02, 435P and 435B cell lines to delineate bone metastasis ability. An improved MCR-ALS model was built using RS after the subtraction of quartz and water signals. Three components were resolved and their respective MCR loadings (left) and scores (right) are plotted. Red triangles: B02, blue squares: 231P, blue crosses: 435B and white diamonds: 435P. Components 1 (A), 2 (B), and 3 (C) have bands compatible with cellular lipids, amino acids and mitochondria-related molecules, respectively. The variance explained for each component is shown as a \%.

Figure 4. MCR analysis of Raman spectra from 231P, B02 (the high bone metastatic cell variant) and B02-PRDX2 that overexpress PRDX2 and have low bone metastasis ability in vivo (Stresing, 2011). Three components were resolved and their respective MCR loadings (left) and scores (right) are plotted. Red triangles: B02, blue squares: 231P, green stars: B02-PRDX2. 
Components 1 (A), 2 (B), and 3 (C) have bands compatible with cellular lipids, amino acids and mitochondria-related molecules, respectively. The variance explained for each component is shown as a $\%$.

Figure 5: A specificity of $100 \%$ and specificity of $90 \%$ is achieved with the use of PLS-DA analysis to discriminate BO2 cells from MDA-MB-435P. This corroborates that RS is able to assess and identify the bone tropism of breast cancer cells.

TABLE

Table 1: Raman Spectra band assignments of MCR components obtained from the MCR-ALS analysis in Fig. 3. Assignments based on references ${ }^{43-55}$.

\begin{tabular}{|l|l||l|l||l|l|}
\hline \multicolumn{2}{|l|}{ Component 1 } & \multicolumn{2}{c||}{ Component 2 } & \multicolumn{2}{c|}{ Component 3 } \\
\hline $\begin{array}{l}\text { Band } \\
\left(\mathrm{cm}^{-1}\right)\end{array}$ & Assignment & $\begin{array}{l}\text { Band } \\
\left(\mathrm{cm}^{-1}\right)\end{array}$ & Assignment & $\begin{array}{l}\text { Band } \\
\left(\mathrm{cm}^{-1}\right)\end{array}$ & Assignment \\
\hline 836 & Saccharide & 693 & Amino acid methionine & 750 & Cytochrome C \\
\hline 1057 & Triacylglycerols & 710 & Amino acid methionine & 777 & Cytosine, Thymine \\
\hline 1075 & Triacylglycerols & 822 & C-C stretch & 804 & Phosphoric acid \\
\hline 1119 & Triacylglycerols & 997 & Phenylalanine & 996 & Phenylalanine \\
\hline 1260 & $\begin{array}{l}\text { Fatty acid, Cholesterol, } \\
\text { Triacylglycerols, } \\
\text { membrane lipids }\end{array}$ & 1026 & Phenylalanine & 1082 & Phospholipids, nucleic acid \\
\hline 1295 & Fatty acid & 1082 & Glutamic acid & 1120 & Cytochrome C \\
\hline 1442 & Fatty acid, Cholesterol & 1122 & C-N proteins & 1203 & Nucleic acid \\
\hline 1660 & Unsaturated fatty acid & 1201 & $\begin{array}{l}\text { Trypt, aromatic C-N and } \\
\text { amide III }\end{array}$ & 1240 & RNA \\
\hline & & 1263 & Amide III & 1312 & Cytochrome C \\
\hline & & 1293 & Alanine & 1333 & Adenine, Guanine \\
\hline & & 1336 & $\begin{array}{l}\text { C-H, Amide III, Trypt, } \\
\text { Glycine }\end{array}$ & 1445 & \\
\hline & & 1442 & CH & 1570 & Cytochrome C, mitochondria \\
\hline & & 1654 & Amide I & 1585 & Cytochrome C, mitochondria \\
\hline
\end{tabular}

\section{REFERENCES}

[1] Samudio, I.; Fiegl, M; Andreeff, M. Cancer R 2009, 69, 2163-2166.

[2] DeBerardinis, RJ.; Mancuso, A.; Daikhin, E; Nissim, I.;Yudkoff, M.; Wehrli, S.; Thomson C. B. PNAS 2007, 104, 1934519350.

[3] Farnie, G.; Sotgia, F.; Lisanti, M. Oncotarget 2015, 6, 30472-30486.

[4] Sotgia, F.; Martinez-Outschoorn, U. E.; Lisanti, M. BMC Medicine 2011, 9,1-5.

[5] Nieva, C.; Marro, M.; Santana-Codina, N.; Rao, S.; Petrov, D.; Sierra, A. PlosOne 2012, 7, 1-10. https://doi.org/10.1371/journal.pone.0046456

[6] Fenn, M. B.; Xanthopoulus, P.; Pyrgiotakis, G.; Grobmyer, S. R.; Pardalos, P. M.; Hench, L. L. Adv. Opt. Techn. 2011, 2011, 120. doi:10.1155/2011/213783

[7] Chan, J.; Taylor, D.; Zwerdling, T.; Lane, S.; Ihara, K. Biophys. J. 2006, 90, 648-656.

[8] Huang, W.; Griffiths, R.; Thompson, I; Bailey, M.; Whiteley, A Anal. Chem. 2004, 76, 4452-4458.

[9] Hedegaard, M.; Krafft, C.; Ditzel, H.; Johansen, L.; Hassing, S.; Popp, J Anal. Chem. 2010, 82, 2797-2802.

[10] Hedegaard, M.; Matthaus, C.; Hassing, S.; Krafft, C.; Diem, M.; J. Popp Theor. Chem. Acc. 2011, 130, $1249-1260$.

[11] Harris, A.; Garg, M.; Yang, X.; Fisher, S.; Kirkham J.; Smith, D.; Martin, D.; High, A. Head Neck Onc. 2009, $38,1-6$.

[12] Balint, S.; Rao, S.; Marro, M.; Miskovsky, P.; Petrov, D. J. Raman Spectrosc. 2010, 42, 1215-1221

[13] Bioucas-Dias, J.; Plaza, A.; Dobigeon, N.; Parente, M.; QuianDu; Gader, P.; Chanussot, J. J. Sel. Topics Appl. Earth Observ.

Remote Sens. 2012, 5, 354-379.

[14] Winter, M. SPIE 1999, 3753, 266-275.

[15] Tauler, R.; Smilde, A.; Kowalski, B. J. Chemometrics 1995, 9, 31-58.

[16] Tu, Q.; Chang, C. Nanomedicine 2012, 8, 545-558.

[17] Bonnier, F.; Knief, P.; Meade, A.; Dorney, J.; Bhattacharya, K. Proc SPIE 2010, 80870F.

[18] Kallaway, C.; Almond, L. M., Barr, H.; Wood, J.; Hutchings, J.; Kendall, C.; Stone, N. Photodiagnosis Photodyn. Ther. 2013, 10, 207-219.

[19] Pence, I.; Mahadevan-Jansen, A. Chem Soc Rev. 2016, 45, 1958-1979.

[20] Kong, K.; Kendall, C.; Stone, N.; Notingher I. Adv. Drug Deliv. Rev. 2015, 89, 121-134.

[21] Azzouz, T.; Tauler, R. Talanta 2008, 74, 1201-1210

[22] Marro, M.; Nieva, C.; Sanz-Pamplona, R; Sierra, A. BBA Mol. Cell. Research 2014, 1843, 1785-1795

[23] Patel, I.; Trevisan, J.; Evans, G.; Llabjani, V.; Martin-Hirsch, P.L.; Stringfellow, H. F.; Martin, F. L. Analyst, 2011, 136, 4950

[24] Marro, M.; Taubes, A.; Abernathy, A.; Balint, S.; Moreno, B.; Sanchez-Dalmau, B.; Martinez-Lapiscina, E. H.; Amat-Roldan, I.; Petrov, D.; Villoslada, P. J Biophotonics, 2014, 7, 724-734.

[25] Olmos, V.; Benitez, L.; Marro, M.; Loza-Alvarez, P.; Piña, B.; Tauler, R.; de Juan, A. TrAC 2017, 94, $130-140$.

[26] Minn, A. J.; Kang, Y.; Serganova, I.; Gupta, G. P.; Giri, D. D.; Doubrovin, M.; Ponomarev, V.; Gerald, W. L.; Blasberg, R.; Massague, J. J Clin Invest 2005, 115, 44-55.

[27] Peyruchaud, O., Serre, C. M.; NicAmhlaoibh, R.; Fournier, P.; Clezardin, P.; J Biol Chem 2003; 278 : $45826-45832$.

[28] Mendez, O., Fernandez, Y., Peinado, M.A.; Moreno, V.; Sierra, A. Clin Exp Metastasis 2005; 22, $297-307$.

[29] Stresing, V.; Baltziskueta, E.; Rubio, N.; Blanco, J.; Arriba, M. C.; Valls, J.; Janier, M.; Clézardin, P.; Sanz-Pamplona, R.; Nieva C.; Marro, M.; Petrov, D.; Sierra, A. Oncogene 2013, 32, 724-735.

[30] Boucharaba, A.; Serre, C.M.; Gres, S.; Saulnier-Blache, J.S.; Bordet, J.C.; Guglielmi ,J.; Clézardin, P.; Peyruchaud, O. J Clin Invest 2004,114, 1714-1725.

[31] Román, I.; Vilalta, M.; Rodriguez, J.; Matthies, A. M.; Srouji, S.; Livne, E.; Hubbell, J. A.; Rubio, N.; Blanco, J. Biomaterials 2007, 28, 2718-2728. 

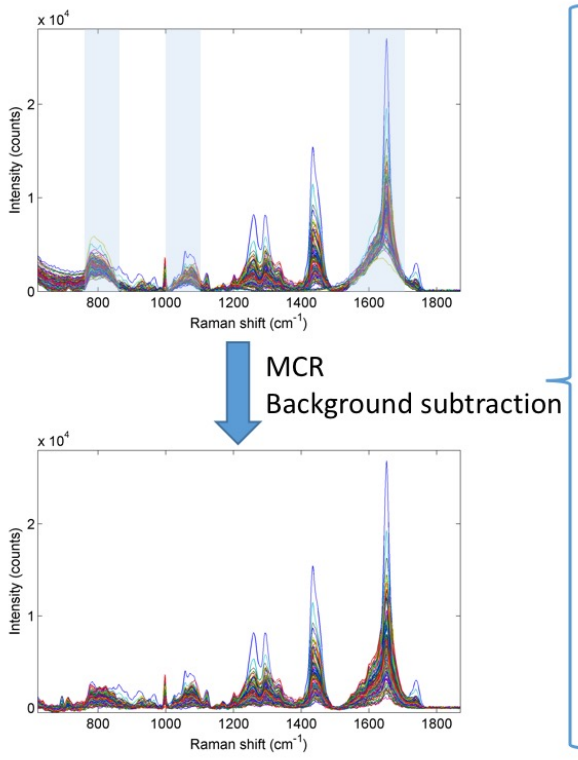

[32] Mikhailyuk, I.; Razzhivin, A. Instr. Exp. Tech 2003, 46, 765-769.

[33] Read, D. Springer 2010

[34] http://lid.phys.msu.su/englishVersion/Science/water.html

[35] Bonnier, F.; Byrne, H. Analyst 2012, 137, 322-332.

[36] Piqueras S.; Duponchel, D.; Tauler, R.; Juan, A. Anal Chim Acta 2011, 705, 182-192.

[37] Huang, C.; Ando, M.; Hamaguchi, H.; Shigeto, S. Anal Chem 2012, 84, 5661-5668.

[38] Hsu, J.; Hsieh, P.; Hsu, H.; Shigeto, S. Sci Rep. 2015, 5, 17541.

[39] Noothalapati, H.; Iwasaki, K.; Yamamoto, T. Anal Sci 2017, 33, 15-22.

[40] Ali, S.; Bonnier, F.; Tfayli, A.; Lambkin, H.; Flynn, K. 2012, J. Biomed. Opt 18, 061202.

[41] Kallaway, C.; Almond, L. M.; Barr, H.; Wood, J.; Hutchings, J.; Kendall, C.; Stone, N. Photodiagnosis Photodyn Ther 2013, $10,207-219$

[42] Andronie, L.; Miresan, V.; Coroian, A.; Pop, I.; Raducu, A.; Rotaru, A.; Coroian, C. O. ProEnvironment 2015, 8, 590-600.

[43] Krafft, C.; Neudert, L.; Simat, T.; Salzer, R. Spectrochim Acta A 2005, 61, 1529-1535.

[44] Zhu, G.; Zhu, X.; Fan, Q.; Wan, X. Spectrochimica Acta Part A 2011,78, 1187-1195.

[45] Notingher I., Sensors 2007, 7, 1343-1358.

[46] Tang, H.; Yao, H.; Wang, G.; Li, Y.; Feng, M. Optics Express 2007, 15, 12708-12716.

[47] Miljkovic, M.; Chernenko, T.; Romeo, M.; Bird, B.; Matthaus, C.; Diem, M. Analyst 2010, 135, 2002-2013.

[48] Adar, F.; Erecinska, M.; Biochemistry 1978, 17, 5484-5488.

[49] Matthaus, C.; Chernenko, T.; Newmark, J.; Warner, C.; Diem, M. Biophys J 2007, 93, 668-673.

[50] Fujita, K.; Smith, I. Mol cells 2008, 28, 530-535.

[51] Okada, M.; Smith, N.; Palonpon, A.; Endo, H.; Kawata, S. PNAS 2012, 109, 28-32.

[52] Hamada, K.; Fujita, K.; Smith, N.; Kobayashi, M.; Inouye, Y.; Kawat, S. J Biom Opt 2008, 13, 0440271- 0440274

[53] Chiu, L.; Ando, M.; Hamaguchi, H. J Raman Spect 2010, 41, 2-3.

[54] Neugebauer, U.; Marz, A.; Henkel, T.; Schmitt, M.; Popp, J. Anal Bioanal Chem 2012, 404, 2819-2829.

[55] Movasaghi, Z.; Rehman, S.; Rehman, I. Applied Spectroc Rev 2007, 42, 493-541.

[56] Wrighta, H. J.; Houb, J.; Xua, B.; Corteza, M.; Potmac, E. O.; Trombergb, B. J.; Razorenova, O. V. PNAS 2017, 114, 65566565.

[57] Jang, Y.; Sharkis, S. Blood 2007, 110, 3056-3063.

[58] Sciacovelli, M.; Gaude, E.; Hilvo, M.; Frezza, C. Methods Enzymol 2014, 542, 1-23.

[59] Bonnet, S.; Archer, S.L.; Allalunis-Turner, J.; Cancer Cell 2007, 11, 37-51.

[60] Sutendra, G.; Michelakis, E. D. Front Onc 2013, 3, 1-11.

[61] Ralser, M.; Wamelink, M.M.; Latkolik, S.; Jansen, E.E.; Lehrach, H.; Jakobs, C. Nat Biotechnol 2009, 27,604-605.

[62] Graves, J.A.; Metukuri, M.; Scott, D.; Rothermund, K.; Prochownik E.V. J Biol Chem 2009, 284: 6520-6529.

[63] Kennecke, H.; Yerushalmi, R.; Woods, R.; Cheang, M.; Voduc, D.; Speers, C. H.; Nielsen, T. O.; Gelmon, K. J Clin Onc 2010, $28,3271-7277$

[64] Minn, A. J.; Gupta, G.P.; Siegel, P.M; Bos, P.D.; Shu, W.; Giri, D.; Viale, A.; Olshen, A.B.; Gerald, W.L.; Massagué, J. Nature 2005 436, 518-524.

[65] Minn, A. J; Gupta, G. P.; Padua, D.; Bos, P.; Nguyen, D.X.; Nuyten, D.; Kreike, B.; Zhang, Y.; Wang, Y.; Ishwaran, H.; Foekens, J. A.; Vijver, M.; Massague, J. PNAS 2007, 104, 6740-5.

[66] Gupta, G.; Nguyen, D.X.; Chiang, A. C.; Bos, P. D.; Kim, J. Y.; Nadal, C. Gomis, R. R.; Manova-Todorova, K.; Massagué, J. Nature 2007, 446, 765-770.

[67] Hug, J.; Hanson, J.; Cheang, M. C.; Nielsen, T. O.; Perou, C. M.; Dumontet, C.; Reed, J.; Krajewska, M.; Treilleux, I.; Rupin, M.; Magherini, E.; Mackey, J.; Martin, M.; Vogel, C. J Clin oncology 2009, 27, 1168-1176.

[68] Martinez-Outschoorn, U. E.; Peiris-Pages, M.; Pestell, R. G.; Sotgia, F.; Lisanti, M.P. Nat. Rev. Clin. Oncol 2017, 14, 11-31. [69] Chowdary, M. V. P; Kumar, K. K.; Kurien, J.; Mathew, S.; Krishna, C. M. Biopolymers 2006 83, 556-569.

[70] Haka, A.; Volynskaya, Z.; Gardecki, J. A.; Nazemi, J.; Lyons, J.; Hicks, D.;Fitzmaurice, M.; Dasari, R. R.; Crowe, J. P.; Feld, M. S. Cancer Research 2006, 66, 3317-3322.

\section{TABLE OF CONTENTS}




\section{Figure 1}
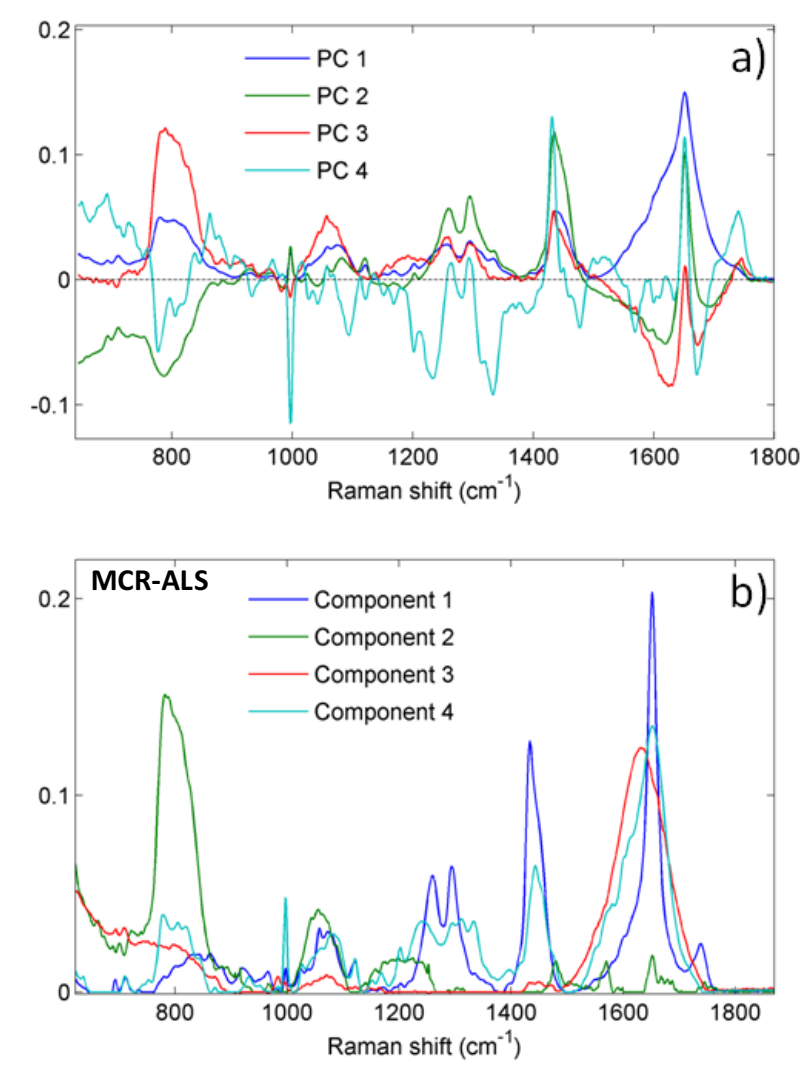

c)

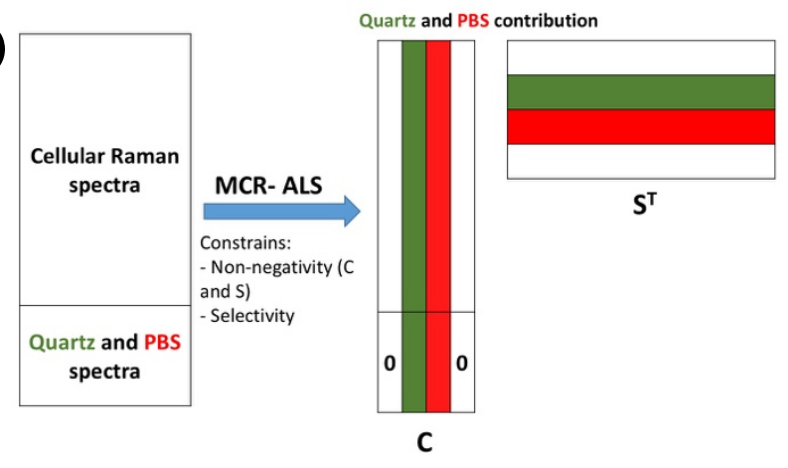




\section{Figure 2}
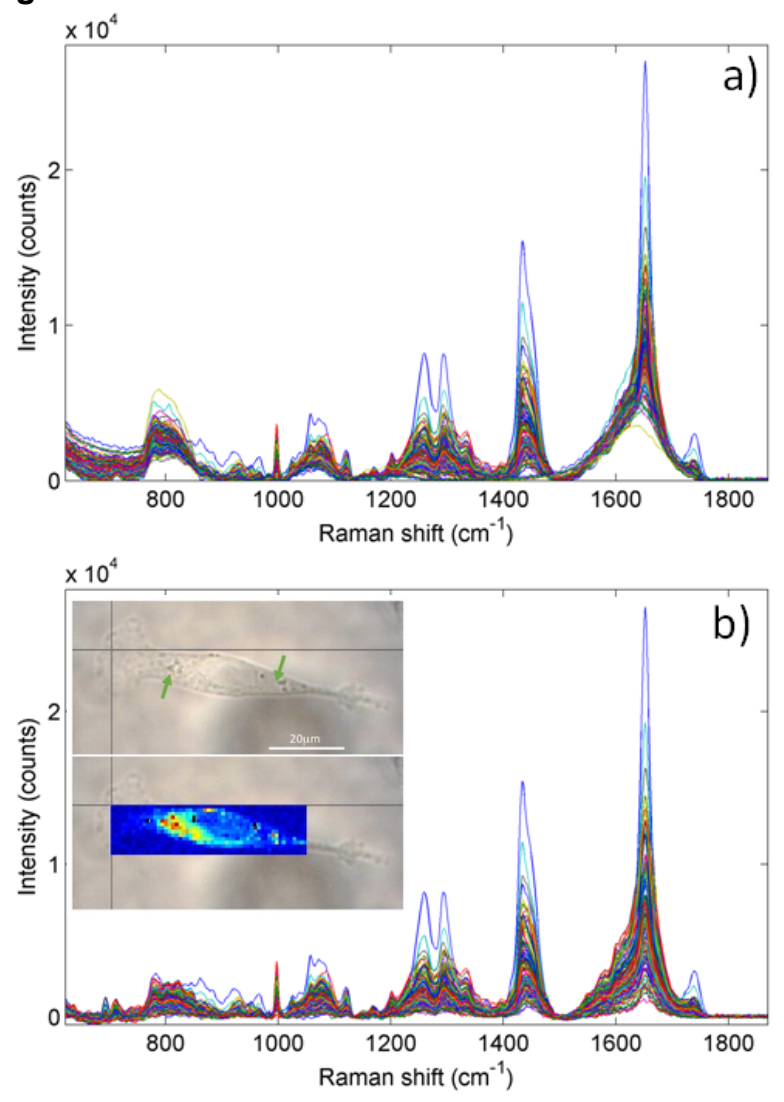

Figure 3

a)
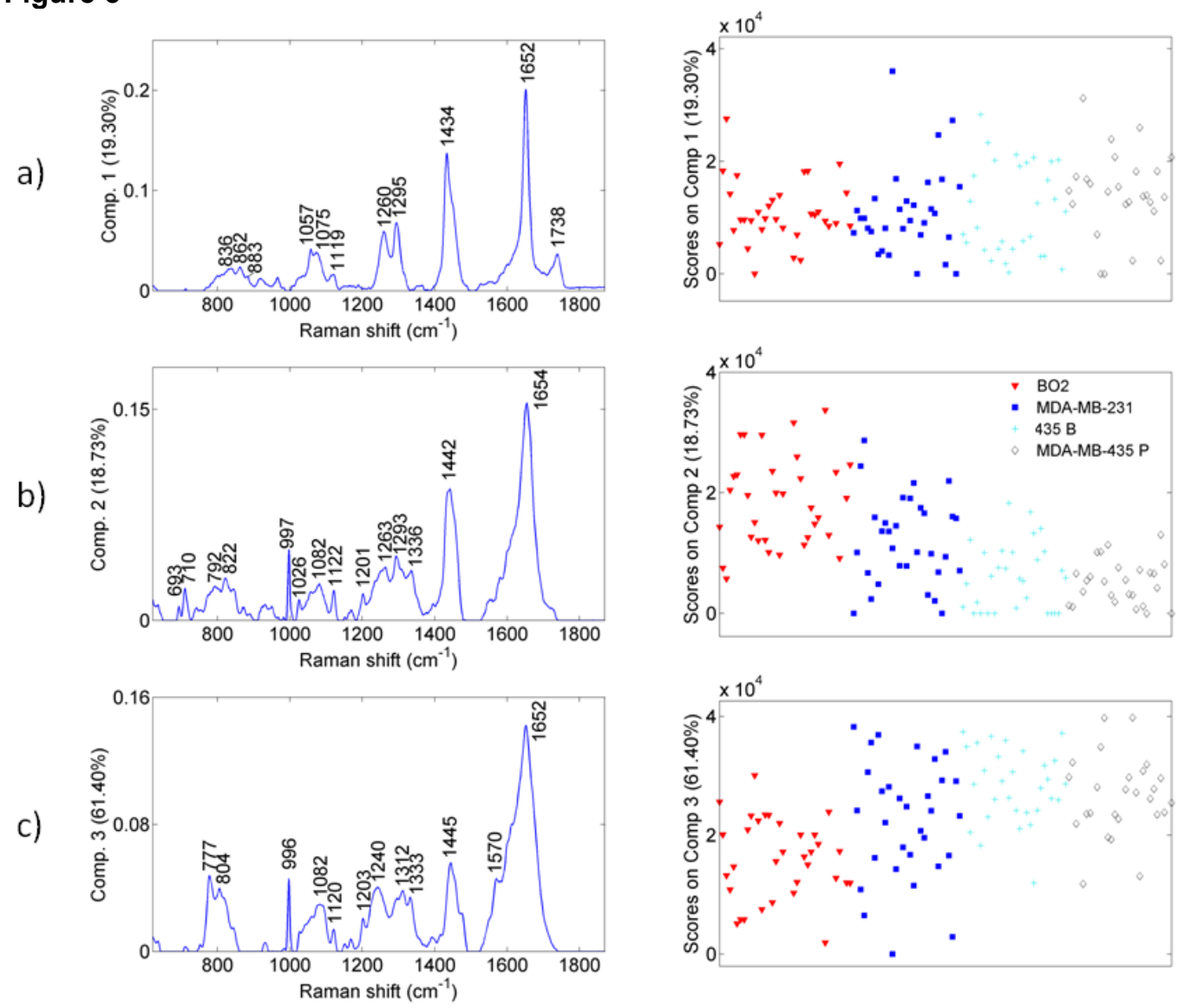
Figure 4

a)
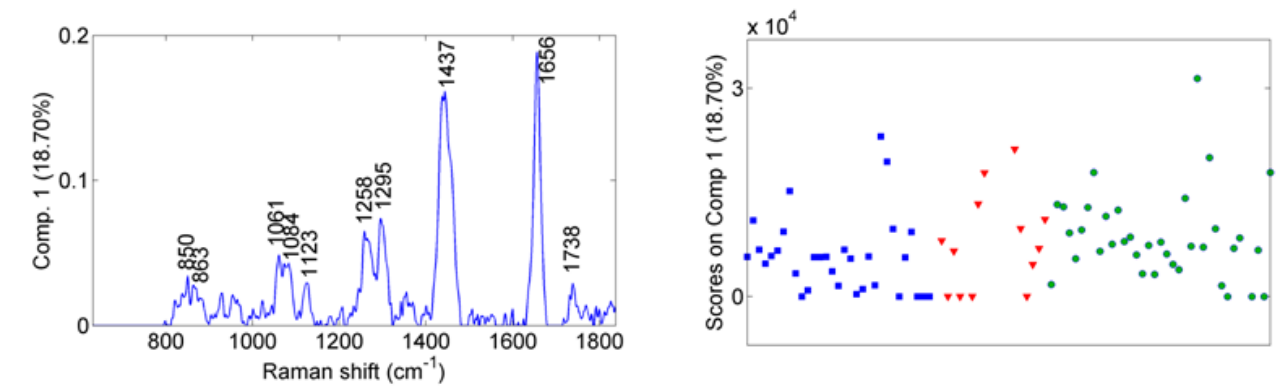

\section{Figure 5}
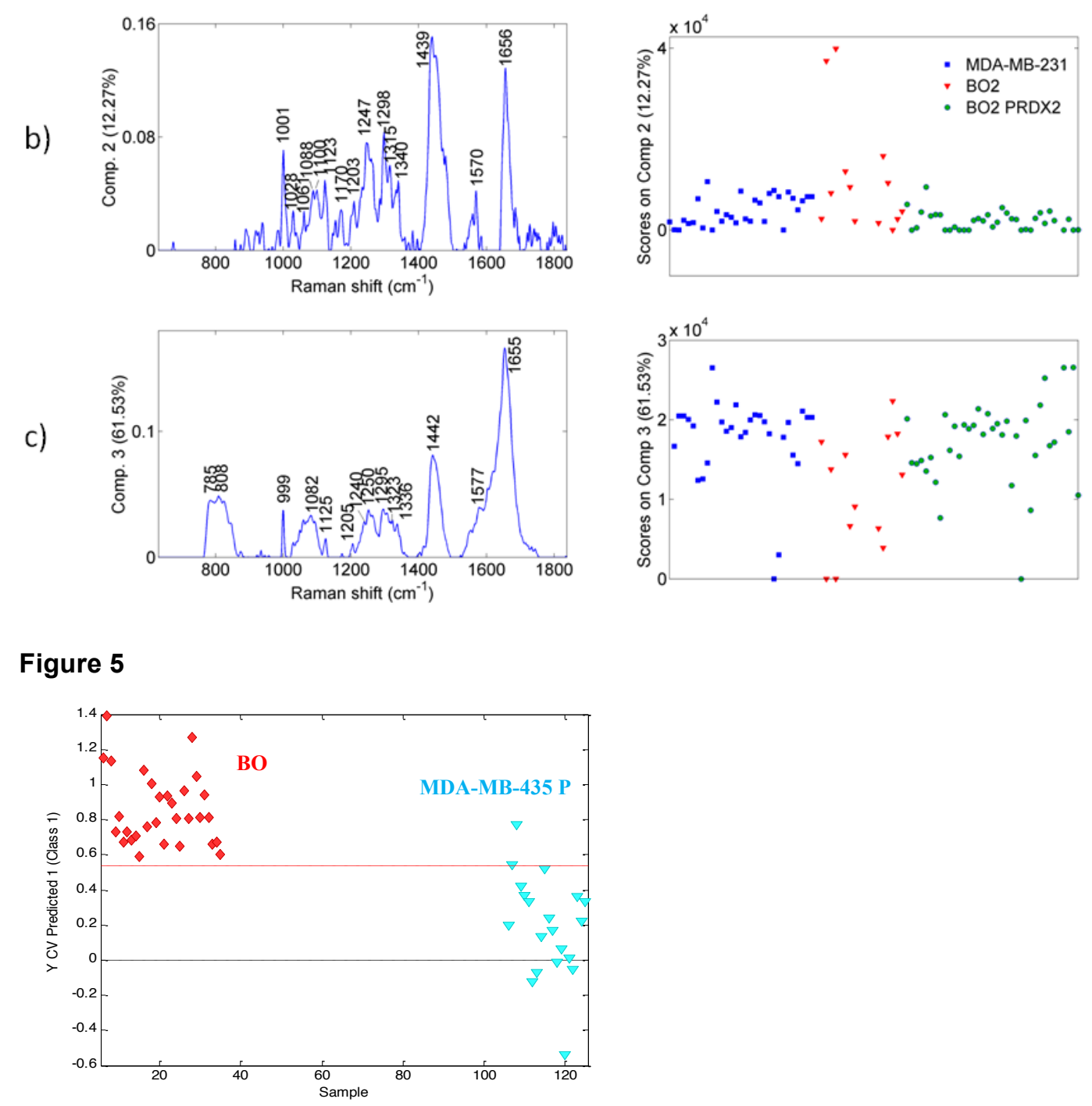


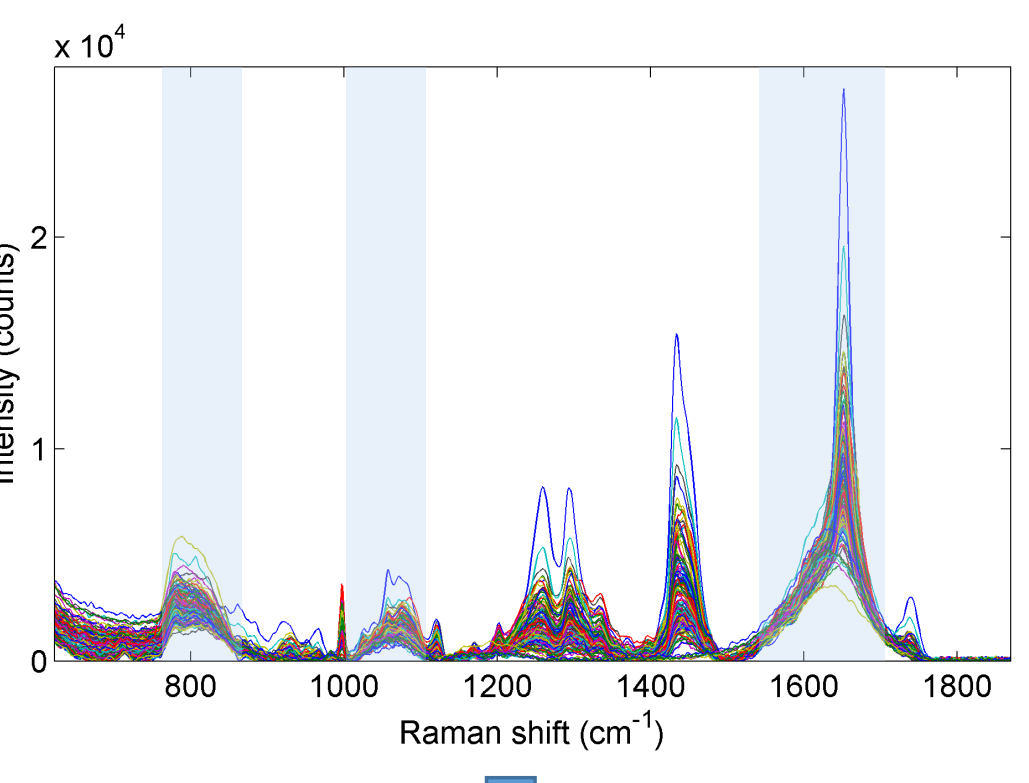

MCR

Background subtraction

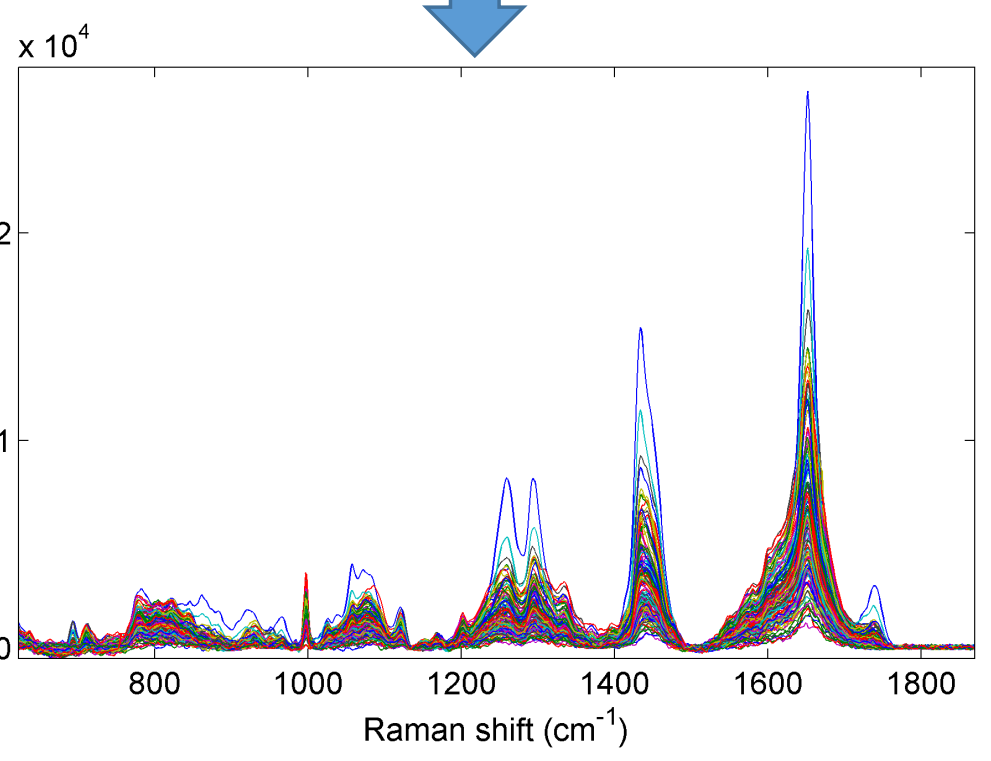

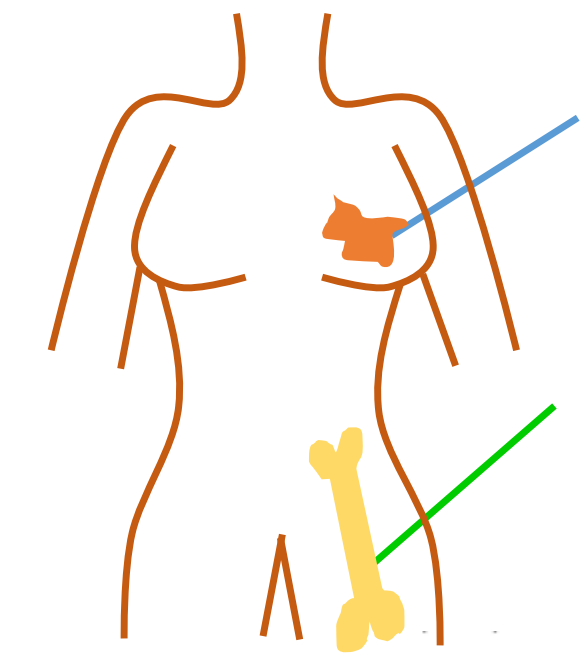

Breast cancer

MDA-MB-231, MDA-MB-435

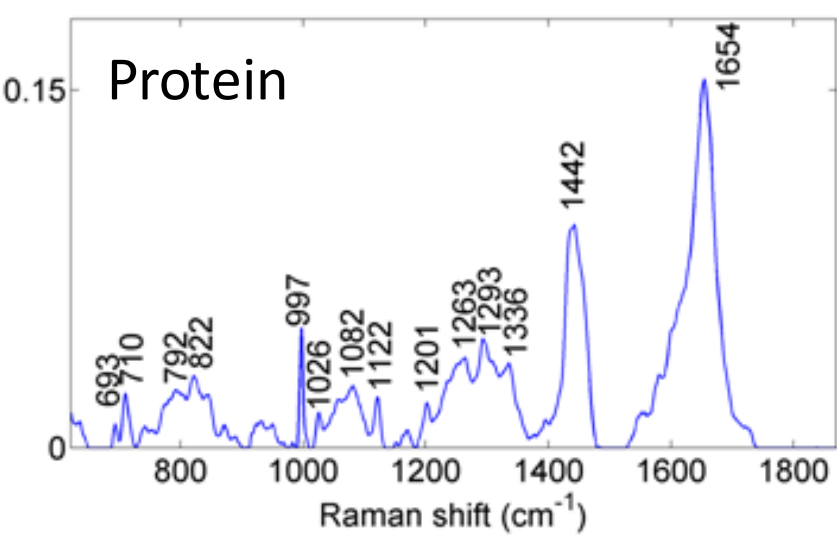

Bone metastasis $\quad \mathrm{BO}$, 435-B

Bone metastasis
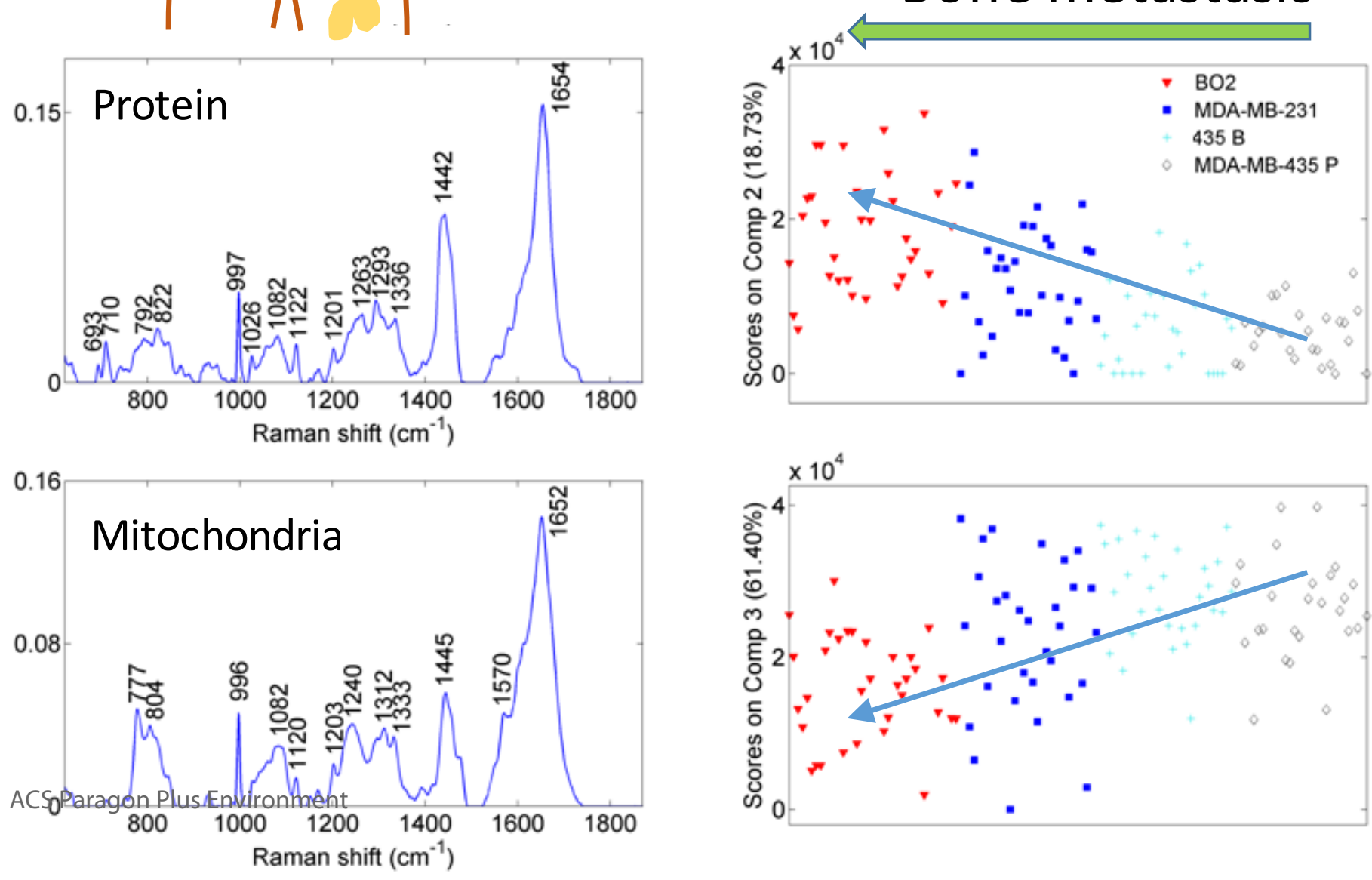